6. Kransdorf MJ, Murphey MD, Sweet DE. Liposclerosing myxofibrous tumor: a radiologic-pathologic-distinct fibro-osseous lesion of bone with a marked predilection for the intertrochanteric region of the femur. Radiology 1999; 212: 693-8.

7. Regado ER, Garcia PBL, Caruso AC, et al. Liposclerosing myxofibrous tumor: a series of 9 cases and review of the literature. J Orthop 2016; 13: 136-9.

8. Deel C, Hassell L. Liposclerosing myxofibrous tumor: a review. Arch Pathol Lab Med 2016; 140: 473-6.

9. Gilkey FW. Liposclerosing myxofibrous tumor of bone. Hum Pathol 1993; 24: 1264

10. Dattilo J, McCarthy EF. Liposclerosing myxofibrous tumor (LSMFT), a study of 33 patients: should it be a distinct entity? Iowa Orthop J 2012; 32: 35 .

11. Ragsdale BD, Sweet DE, Vinh TN. Radiology as gross pathology in evaluating chondroid lesions. Hum Pathol 1989; 20: 930-51.

DOI: https://doi.org/10.1016/j.pathol.2020.09.032

\section{A NUT carcinoma lacking squamous differentiation and expressing TTF1}

Sir,

NUT carcinoma (NC) is a rare highly aggressive malignancy refractory to most therapies, with a median overall survival of less than a year. ${ }^{1} \mathrm{NC}$ often shows keratinising foci and typically expresses high molecular weight cytokeratins, p63 and p40 $(\Delta \mathrm{Np} 63)$ by immunohistochemistry (IHC), and therefore is considered to be a poorly differentiated type of squamous cell carcinoma. ${ }^{2}$

Despite the near universal poor outcome, timely diagnosis of NC is important for appropriate clinical management and counselling. The presence of a fusion between the nuclear protein in testis (NUT or NUTMI) gene and bromodomain (BRD) and extra terminal domain protein family (BET) members $B R D 4$ or $B R D 3$ is considered pathognomonic, but in practise, expression of NUTM1 protein by IHC is often regarded as diagnostic. ${ }^{2}$ However, expression of NUTM1 and/or NUTM1 rearrangements can be present in tumours that are differential diagnoses for $\mathrm{NC}$, including some germ cell tumours, lymphomas and sarcomas. ${ }^{3}$ Here we report an atypical TTF1/NUTM1-expressing malignancy that lacked evidence of squamous differentiation and required RNA sequencing in order to identify a BRD4-NUTM1 fusion transcript to make a definitive diagnosis of NC.

A 22-year-old male, a non-smoker, presented with a one month history of dyspnoea and nausea. Imaging showed extensive mediastinal, nodal and bone disease. A piecemeal biopsy from the mediastinal mass and a transbronchial needle aspirate (TBNA) from subcarinal lymph nodes showed sheets of malignant cuboidal cells with indistinct cytoplasmic borders, pale eosinophilic vacuolated cytoplasm, large irregular nuclei, vesicular chromatin and large nucleoli. No keratin or inter-cellular bridges or glandular differentiation were identified. Malignant cells were also present in a pleural fluid specimen.

IHC (Fig. 1) on all three specimens showed weak cytoplasmic expression of CAM5.2 and strong diffuse nuclear expression of TTF1. PAX8, MYC, SOX2 and PRAME (a melanocytic marker) were also positive in tumour cells.
Tumour cells in the mediastinal biopsy showed unusual granular cytoplasmic membrane pattern of expression of pancytokeratin AE1/AE3. AE1/AE3 immunostains were negative in tumour cells in the TBNA and pleural fluid specimens. IHC for the squamous cell markers p63, p40 and cytokeratins CK5/6, CK1/5/10/14 (clone 34ßE12) were negative in all specimens, as were CK7, CK19, CK20, napsin-A, CD56, synaptophysin, S100, SOX10, vimentin, germ cell (SALL4, OCT3/4, KIT, PLAP, CD30) and lymphoid markers (CD45, CD3, CD5, CD19, CD20, BCL6, TdT).

IHC for NUTM1 showed speckled nuclear staining in tumour nuclei (Fig. 1) and NUTM1 (chromosome 15q14) breakapart fluorescent in situ hybridisation (FISH) showed split signals in tumour cells (not shown). RNA was extracted from formalin fixed, paraffin embedded (FFPE) tumour from the mediastinal biopsy. Whole transcriptome sequencing was performed with the Illumina NextSeq 550 platform (Illumina, USA). An in-frame RNA fusion transcript involving exon 14 of BRD4 and exon 3 of NUTM1 (Fig. 2A) was identified by two fusion calling programs (Arriba ${ }^{4}$ and Pizzly ${ }^{5}$ ) that are based on unrelated algorithms. Based on the clinical, histological and molecular findings, a diagnosis of NC was made despite the lack of squamous differentiation.

The transcriptome of the tumour was investigated further by comparing it to a transcriptome obtained from RNA sequencing FFPE tissue from a NC with a BRD3-NUTMI fusion. Although this fusion is present in only $\sim 10 \%$ of $\mathrm{NC}$, this tumour showed typical histology and protein expression by IHC for a NC, i.e., strong expression of p40 and high molecular weight cytokeratins, consistent with squamous differentiation. It did not express TTF1. Normalised RNA expression profiles from both NCs showed similar levels of NUTM1 (Fig. 2). Of the known BRD-NUTMI transcriptional targets, MYC, TP63 and $S O X 2^{6,7}$ high expression of $M Y C$ was seen in both NC, TP63 was only highly expressed in the typical NC and SOX2 in the BRD4-NUTM1 NC (Fig. 2B). There were high RNA levels for multiple cytokeratins including KRT4, KRT5, KRT7, KRT14, KRT17 and KRT19 in the typical NC, but not in the BRD4-NUTMI NC. Cytokeratins with detectable RNA in the BRD4-NUTM1 NC were $K R T 8$ and KRT18. RNAs that were highly expressed in the BRD4-NUTM1 NC included NKX2-1 (the gene for TTF1), PRAME and PAX6. BCL6 RNA was highly expressed in the typical NC but not BRD4-NUTM1 NC.

Overall, the transcriptome findings were consistent with our IHC findings. There were high RNA and protein levels for TTF1/NKX2-5 in the BRD4-NUTM1 NC, and low RNA and protein expression levels for p63, p40, BCL6 and most cytokeratins, with the exception of CK8 and CK18 (notably CAM5.2 and AE1/AE3 both detect CK8; expression of CK18 was unable to be confirmed due to exhaustion of tumour tissue). $P A X 6$, but not $P A X 8$, RNA was expressed at high levels in the BRD4-NUTM1 NC. However, PAX8 was readily detectable by IHC in the BRD4-NUTMI NC. PAX6 IHC was not performed, and it is possible that cross reaction of the PAX8 clone that we used (MRQ-50) with PAX6 may explain the discordance between the RNA and IHC results, although this was not investigated further.

The diagnosis of $\mathrm{NC}$ is often relatively straightforward, as the clinical presentation, morphology and immunophenotype in most cases is typical. ${ }^{2}$ NUTM1 IHC shows a near $100 \%$ 

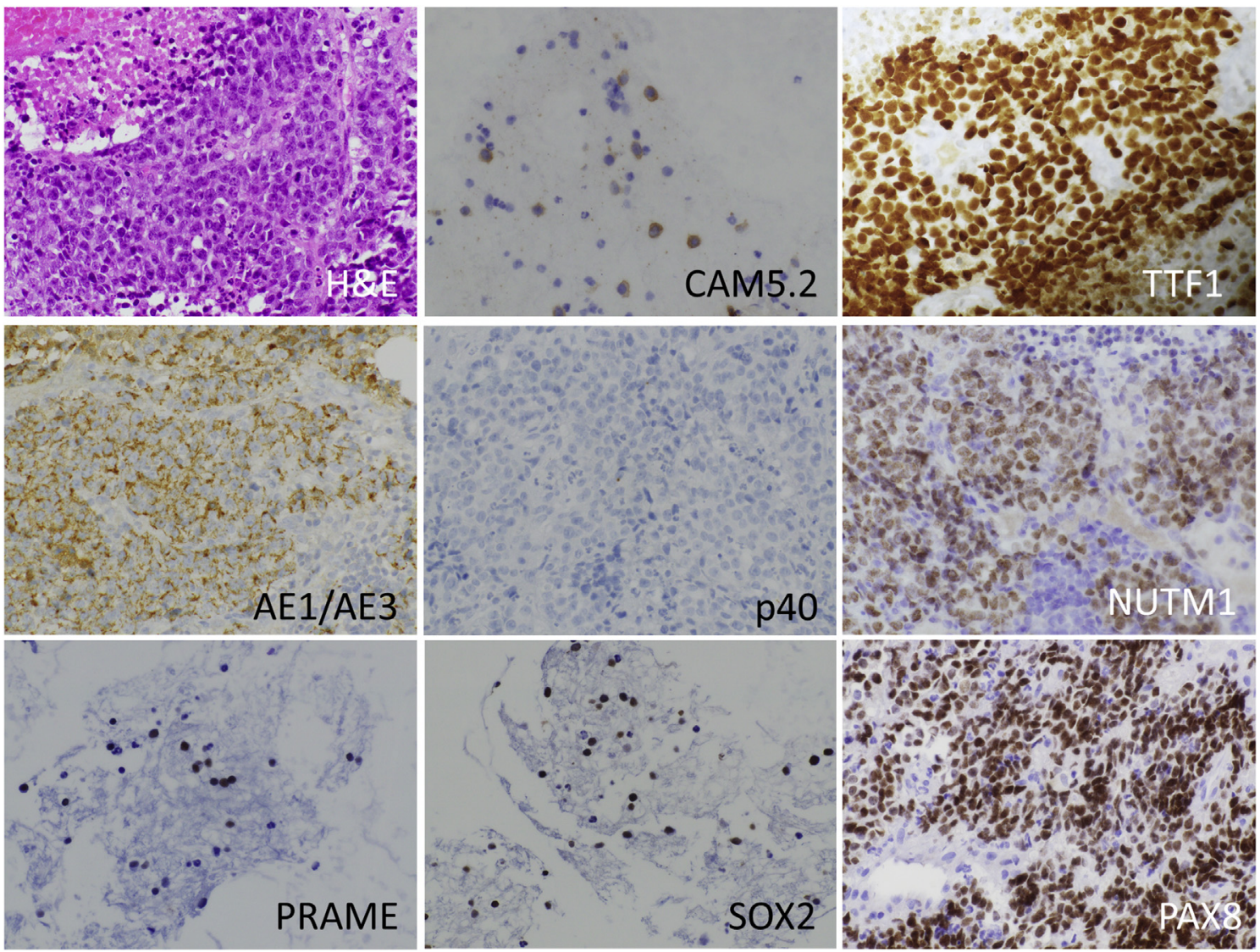

Fig. 1 Morphology and immunohistochemistry of the BRD4-NUTM1 carcinoma in biopsies from mediastinum mass (H\&E, TTF1, AE1/AE3, p40, NUMT1, PAX8), subcarina TBNA (CAM5.2) and pleural fluid (PRAME, SOX2).

sensitivity for $\mathrm{NC}$ and therefore is a cheap and rapid method to confirm diagnosis in such circumstances. Recognised challenges to the diagnosis of $\mathrm{NC}$ include consideration in older patients, in atypical tumour locations (e.g., nonmidline, non-head/neck/mediastinal), ${ }^{8}$ and in tumours with variant morphologies including lack of keratinising foci, a particular issue in small biopsies, as well as occasionally pseudo-glandular, rhabdoid and even chondroid differentiation. $^{3}$ In these scenarios the diagnosis can be missed if NUTM1 IHC is not performed.

The NC presented here showed a typical clinical presentation and tumour morphology despite the lack of keratinising foci, and expressed NUTM1. However, the lack of p63/p40 and high molecular weight cytokeratin expression was atypical for $\mathrm{NC}$ and raised the possibility of other diagnoses. Seminoma and embryonal carcinoma can occasionally express NUTM1 in the absence of a NUTM1 rearrangement. ${ }^{9}$ Acute lymphoblastic leukaemia (ALL) and some rare sarcomas with small round, spindle and/or rhabdoid cell morphologies can also show NUTM1 expression and harbour NUTM1-rearrangements. ${ }^{3}$ In NUTM1-rearranged sarcomas, NUTM1 is not fused to a $B E T$ gene but to other genes including members of the MAX dimerisation (MAD) family or the capicua transcriptional repressor $(C I C)$. In the present case extensive IHC, NUTM1 FISH and RNA sequencing effectively excluded these possibilities.
BRD4-NUTM1 is the most common NC associated fusion, being present in $70-80 \%$ of cases. ${ }^{2}$ Fusion of exon 11 of $B R D 4$ to exon 3 of NUTM1 appears to be most frequent chimeric RNA transcript in NC. We considered the possibility that the additional exons $12-14$ of $B R D 4$ in this case may have contributed to the atypical RNA and protein expression. However, the fusion of BRD4 exon 14 to NUTM1 exon 3 has been reported multiple times, including in p63expressing NC. ${ }^{10}$

The combination of strong TTF1 expression and absent squamous differentiation appears to be novel in NC. However, due to the paucity of reported TTF1 IHC data, the real incidence may under estimated. In a multi-institution cohort of $21 \mathrm{NC}^{11}$ harbouring NUTM1 fusions with either BRD4 (18 cases), BRD3 (1 case) or NSD3 (3 cases), where reported, markers of squamous differentiation (p63, p40 and/or high molecular cytokeratin) were absent in $2 / 17$ cases $(12 \%)$, and TTF1 was weakly or patchily expressed in $2 / 8(25 \%)$ cases. Both TTF1-positive NC harboured BRD4-NUTM1 fusions, but in contrast to the NC that we report here, also expressed p63 and CK5/6. We identified only one other report in which 'diffuse moderate' TTF1 expression as well as p63, CK5 and high molecular weight cytokeratins (CK1/5/10/14) expression was noted in a NC. ${ }^{12}$ In a series of six soft tissue/visceral tumours with NUTM1 fusions, ${ }^{8}$ two small round cell tumours with BRD4-NUTM1 fusions were described that are somewhat similar to the tumour described here in that they lacked 


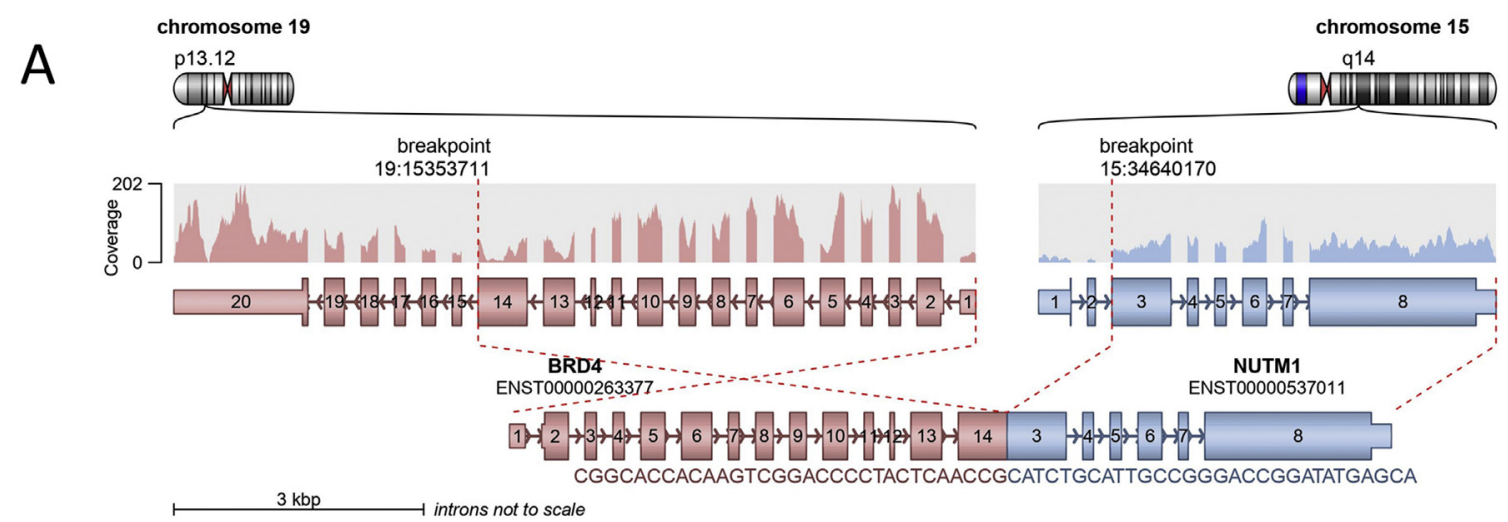

B

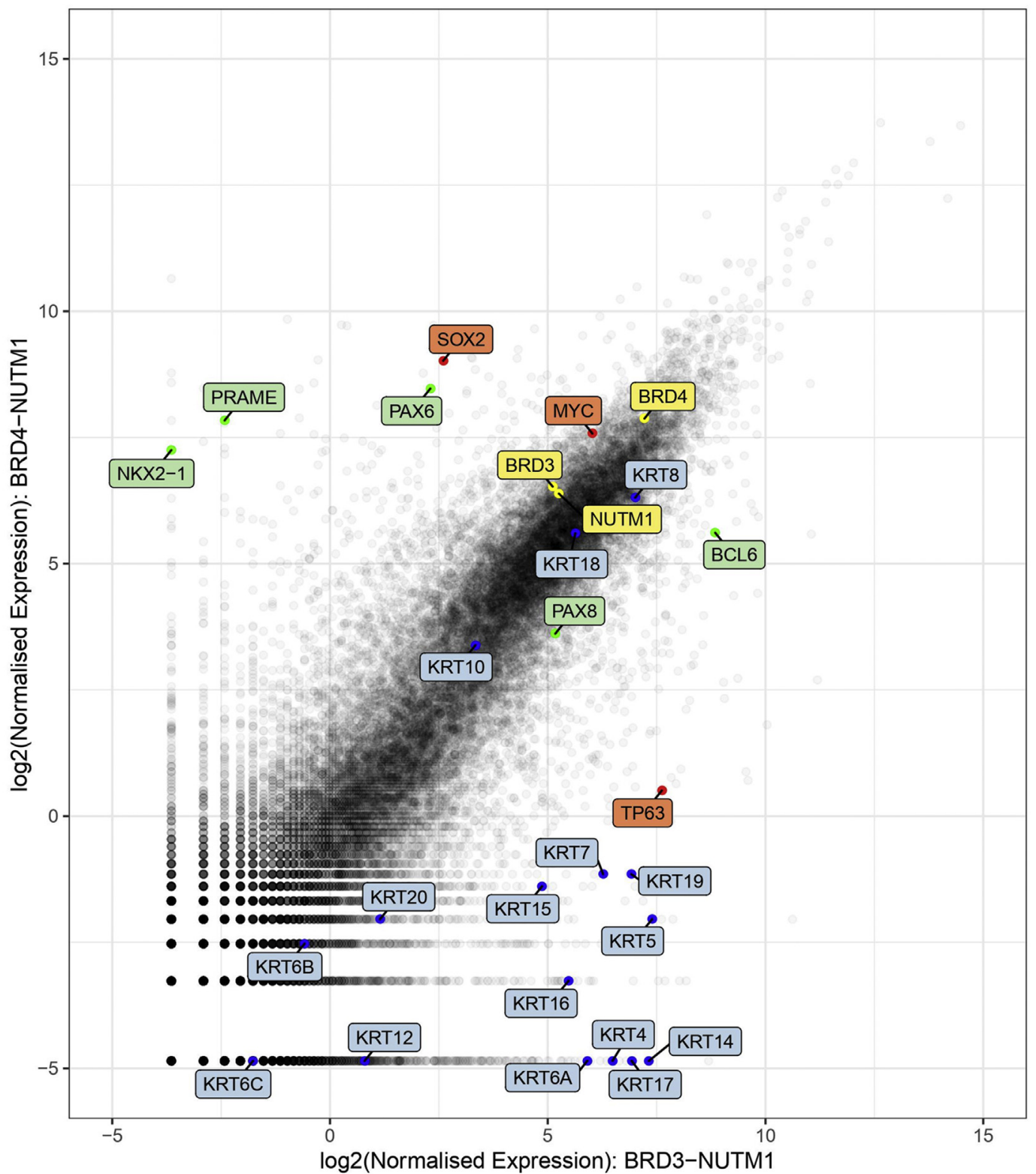

Fig. 2 (A) BRD4-NUTM1 fusion between BRD4 exon 14 (NM_058243.2) and NUTM1 exon 3 (NM_175741.2) detected by RNA whole transcriptome analysis. (B) Scatterplot of normalised RNA expression in the BRD4-NUTM1 NC compared to a BRD3-NUTM1 NC. Genes thought to be critical for oncogenesis in NC (MYC, TP63 and $S O X 2$ ) are labelled orange, keratins blue, genes involved in the fusions yellow, and other genes discussed in the text green. 
keratinising foci and had weak or absent cytokeratin and/or p63 expression. TTF1 expression was not reported in these tumours.

Together with this case, these studies suggest that it may not be uncommon for NC to show either TTF1 expression, or lack squamous differentiation. This may be an important potential source of misdiagnosis. NC with TTF1 expression could be erroneously classified as either lung adenocarcinoma or thyroid carcinoma. NC lacking expression of squamous markers and weak/negative low molecular weight cytokeratin expression may be classified as a sarcoma, a carcinoma of unknown primary or an undifferentiated malignancy. The likelihood of such errors would be compounded for NC with an atypical clinical presentation or variant tumour morphology, as in such cases NUTM1 IHC is less likely to be performed. For these reasons it would seem judicious to have a low threshold for screening high grade or poorly differentiated malignancies by NUTM1 IHC or FISH, and in positive cases to perform RNA sequencing to identify the specific NUTM1 fusion partner in order to confirm a diagnosis of $\mathrm{NC}$.

Acknowledgements: The authors thanks Prof Anthony Gill (Department of Anatomical Pathology, Royal North Shore Hospital) and Dr Michael Christie (Royal Melbourne Hospital, Melbourne Health Shared Pathology Service) for donation of tissue. Tumour profiling at Peter Mac is made possible by the generous support of the Peter MacCallum Foundation.

Conflicts of interest and sources of funding: The authors state that there are no conflicts of interest to disclose.

\section{Owen W. J. Prall ${ }^{1}$, Niko Thio ${ }^{2}$, Satwica Yerneni ${ }^{1}$, Beena Kumar ${ }^{3}$, Christopher R. McEvoy ${ }^{1}$}

${ }^{1}$ Department of Pathology, Peter MacCallum Cancer Centre, Melbourne, Vic, Australia; ${ }^{2}$ Cancer Research Division, Peter MacCallum Cancer Centre, Melbourne, Vic, Australia; ${ }^{3}$ Monash Health Pathology, Monash Medical Centre, Clayton, Australia

Contact Dr Owen W. J. Prall.

E-mail: owen.prall@petermac.org

1. Bauer DE, Mitchell CM, Strait KM, et al. Clinicopathologic features and long-term outcomes of NUT midline carcinoma. Clin Cancer Res 2012; 18: 5773-9.

2. French CA. NUT Carcinoma: clinicopathologic features, pathogenesis, and treatment. Pathol Int 2018: 68: 583-95.

3. McEvoy CR, Fox SB, Prall OWJ. Emerging entities in NUTM1 rearranged neoplasms. Genes Chromosom Cancer 2020; 59: 375-85.

4. Uhrig S, Fröhlich M, Hutter B, Brors B. Arriba - fast and accurate gen fusion detection from RNA-Seq data. ESMO Open 2018; 3 (Suppl 2). $\mathrm{PO}-400$

5. Melsted P, Hateley S, Joseph IC, et al. Fusion detection and quantification by pseudoalignment. bioRxiv 2017; 20: 166322.

6. Wang R, Liu W, Helfer CM, et al. Activation of SOX2 expression by BRD4-NUT oncogenic fusion drives neoplastic transformation in NUT midline carcinoma. Cancer Res 2014; 74: 3332-43.

7. Alekseyenko AA, Walsh EM, Wang X, et al. The oncogenic BRD4 NUT chromatin regulator drives aberrant transcription within large topological domains. Genes Dev 2015; 29: 1507-23.

8. Dickson BC, Sung YS, Rosenblum MK, et al. NUTM1 gene fusions characterize a subset of undifferentiated soft tissue and visceral tumors. Am J Surg Pathol 2018; 42: 636-45.
9. Haack H, Johnson LA, Fry CJ, et al. Diagnosis of NUT midline carcinoma using a NUT-specific monoclonal antibody. Am J Surg Pathol 2009: 33: 984-91.

10. Minato H, Kobayashi E, Nakada S, et al. Sinonasal NUT carcinoma: clinicopathological and cytogenetic analysis with autopsy findings. Hum Pathol 2018; 71: 157-65.

11. Stevens TM, Morlote D, Xiu J, et al. NUTM1-rearranged neoplasia: a multi-institution experience yields novel fusion partners and expands the histologic spectrum. Mod Pathol 2019; 32: 764-73.

12. Tanaka M, Kato K, Gomi K, et al. NUT midline carcinoma: report of 2 cases suggestive of pulmonary origin. Am J Surg Pathol 2012; 36 $381-8$.

DOI: https://doi.org/10.1016/j.pathol.2020.09.027

\section{Keratosis pilaris with adjacent haemosiderin deposition: a clue to scurvy}

Sir,

We present the case of a 50-year-old man with a misleading 'classical' presentation and the histological assessment which was the key to his diagnosis. This patient developed a purpuric rash several weeks prior to his hospital presentation. The rash arose in his lower limbs which rapidly progressed proximally with increasing generalised pain. It was non-blanching, confluent, palpable and purpuric in nature. Given that the clinical picture resembled typical features of a vasculitic rash, extensive laboratory tests including haematological, biochemical and immunological tests were performed. A biopsy of the rash was undertaken which marked a critical turning point in his clinical course.

Two skin punch biopsies were received for histological assessment. Both biopsies showed a superficial perivascular lymphocytic infiltrate. In one biopsy, keratosis pilaris with perifollicular haemosiderin deposition was present (Fig. 1AD). No vasculitis was seen and there were no extravasated red blood cells. Perls stain (used to highlight iron) was performed which demonstrated perifollicular haemosiderin deposition (Fig. 1E,F). The combination of keratosis pilaris and perifollicular haemosiderin deposition raised the strong possibility of scurvy. The histology result was conveyed back to the treating clinicians which prompted the investigation of scurvy.

A review of systems revealed that the patient had not consumed fresh vegetables or fruits for the past 10 years. He did not eat regularly and had been consuming large amounts of alcohol since his employment had been terminated. Further examination revealed gingival bleeding and ecchymosis. His body mass index was borderline underweight at $18.9 \mathrm{~kg} / \mathrm{m}^{2}$ and he had lost $16 \mathrm{~kg}$ ( $21 \%$ weight loss) in the preceding 2 years. This patient's serum ascorbate (vitamin C) level was indeed low at $9 \mu \mathrm{mol} / \mathrm{L}$ (normal range $20-120 \mu \mathrm{mol} / \mathrm{L}$ ), further supporting the diagnosis of scurvy. The diagnosis of vasculitis was dismissed, and a nutritional screen and supplementation were undertaken. Unfortunately, dermatological specialty opinion was not sought nor was a comprehensive skin examination performed, which may have revealed some of the key cutaneous findings of scurvy as discussed below. 